\title{
Características evolutivas do Cysticercus cellulosae no encéfalo e no coração humanos
}

\author{
Developmental characteristics of Cysticercus cellulosae in the human brain and heart
}

Ruy de Souza Lino Júnior ${ }^{1}$, Patrícia M. Ribeiro², Élida Juliana Antonelli², Ana Carolina G. Faleiros², Sílvia A. Terra ${ }^{2}$, Marlene A. dos Reis ${ }^{2}$ e Vicente de Paula A. Teixeira ${ }^{2}$

\begin{abstract}
Resumo O presente estudo teve como objetivos avaliar a prevalência de cisticercose, classificar as etapas evolutivas dos cisticercos encontrados nos encéfalos e corações humanos, diferenciá-las de acordo com os aspectos macro e microscópicos dos processos patológicos gerais e comparar os processos encontrados nos encéfalos e corações. Foram revisados protocolos de autópsias realizadas no Hospital Escola da Faculdade de Medicina do Triângulo Mineiro, Uberaba, MG, Brasil, no período de 1970 a 2000. Verificou-se a prevalência da cisticercose em 71 casos, sendo $18(25,4 \%)$ de cisticercose cardíaca e $53(74,6 \%)$ de encefálica. Foram analisados 19 cisticercos, sendo 9 de encéfalos e 10 de corações. Os cisticercos foram classificados, de acordo com sua fase evolutiva, em etapas: vesicular, vesicular coloidal, granular nodular e nodular calcificada, com similaridade entre os diagnósticos macro e microscópico. Entre os processos patológicos destacaram-se a beta-fibrilose e a fibroelastose endocárdica. Adicionalmente, demonstrou-se que a classificação pode ser aplicada tanto na cisticercose encefálica como na cardíaca.
\end{abstract}

Palavras-chaves: Cisticercose cardíaca. Classificação da cisticercose. Neurocisticercose. Beta-fibrilose.

Abstract The present study aimed to evaluate the prevalence of cysticercosis, to classify the developmental phases of cysticerci found in human brains and hearts, and differentiate these according to the macro and microscopic aspects of the general pathological processes, and to compare the process found in the brains and hearts. Protocols from autopsies performed at the Hospital of the School of Medicine of the Triângulo Mineiro, Uberaba, MG, Brazil, in the period from 1970 to 2000 were reviewed. The prevalence of cysticercosis was verified in 71 cases, of which $53(74.6 \%)$ were encephalic cysticercosis and $18(25.3 \%)$ cardiac cysticercosis. Nineteen cysticerci were analyzed, from 9 brains and 10 hearts. The cysticerci were classified according to their developmental stage: vesicular, colloidal vesicular, granular nodular and calcified nodular, with similarities between the macroscopic and microscopic diagnoses. Among the pathological processes found beta-fibrilosis and endocardial fibroelastosis are underscored. In addition, it was demonstrated that this classification may be applied both to encephalic and cardiac cysticercosis.

Key-words: Cardiac cysticercosis. Classification of cysticercosis. Encephalic cysticercosis. Beta-fibrilosis.

A teníase e a cisticercose são afecções produzidas pelas fases adultas e larvárias de helmintos da família Taenidae. Existem duas espécies que afetam humanos (Taenia solium e T. saginata), que necessitam de dois hospedeiros distintos para completarem seus ciclos de vida. Os humanos são os hospedeiros definitivos para o estado adulto de ambas as espécies de tênias, enquanto os suínos e bovinos são os hospedeiros intermediários para os estágios larvários desses parasitas $^{1123}$.
Os humanos, bovinos ou suínos se infectam ao ingerirem os ovos dos parasitas presentes em alimentos contaminados. Esses ovos atingem o segmento intestinal e sofrem ação das enzimas gástricas e pancreáticas, que digerem o embrióforo liberando o embrião hexacanto ou oncosfera. O embrião, ao penetrar na mucosa, sofre disseminação linfohematogênica alojando-se em diferentes tecidos dos vários órgãos do organismo ${ }^{11} 142223$, sendo mais freqüente se alojarem no sistema nervoso central e no

\footnotetext{
1. Disciplina de Patologia Geral do Instituto de Patologia Tropical e Saúde Pública da Universidade Federal de Goiás, Goiânia, GO. 2. Disciplina de Patologia Geral do Departamento de Ciências Biológicas da Faculdade de Medicina do Triângulo Mineiro, Uberaba, MG.

Suporte financeiro: Fundação de Ensino e Pesquisa de Uberaba (FUNEPU), Fundação de Amparo à Pesquisa do Estado de Minas Gerais (FAPEMIG) e Conselho Nacional de Desenvolvimento Científico e Tecnológico (CNPq).

Endereço para correspondência: Prof. Vicente de Paula Antunes Teixeira. Disciplina de Patologia Geral/FMTM. R. Frei Paulino 30, Bairro Abadia, 38025-180, Uberaba, MG, Brasil.

Tel.: 5534 3318-5428, Fax: $55343312-6640$

email: vicpat@dcb.fmtm.br

Recebido para publicação em 22/2/2002
} 
músculo esquelético ${ }^{11}$, provocando uma reação hospedeiro-parasita com ativação dos mecanismos de defesa e desenvolvimento de resposta inflamatória ${ }^{13}$.

Há um polimorfismo clínico, pois as manifestações são inespecíficas e variam de acordo com o local de infecção, com o número e características das lesões, com a fase de desenvolvimento do parasita e com a intensidade da resposta imune-inflamatória do hospedeiro frente ao cisticerco ${ }^{212834}$.

A cisticercose, raramente, é a causa de morte de pacientes adultos autopsiados, porém tem um impacto sócio-econômico significante, tanto pela inaptidão temporária ou permanente que ocasiona em indivíduos em idade produtiva, quanto pelo seu alto custo em relação a diagnóstico e tratamento ${ }^{21}$.

O cisticerco é considerado viável quando está na etapa vesicular (EV), isto é, com uma membrana transparente contendo líquido e a larva invaginada em seu interior, sendo que há áreas de tegumento do parasita histologicamente intactas, e outras áreas com infiltrado inflamatório no hospedeiro e discreta gliose. Nessa primeira etapa, a resposta imune pode variar de tolerância até intensa resposta inflamatória. A próxima etapa é a vesicular coloidal (EVC), que caracteriza-se por necrose da larva e a vesícula aparece mais espessa e com líquido turvo ou fracamente gelatinoso, esbranquiçado. O escólex apresenta sinais de degeneração hialina. No tegumento do parasita observam-se alterações, tais como, infiltrado inflamatório com linfócitos, neutrófilos, monócitos e células gigantes do tipo corpo estranho, além de fibroblastos e fibras colágenas. No tecido do hospedeiro, as arteríolas vizinhas ao parasita apresentam endarterite e necrose fibrinóide na túnica média, encontrando-se, eventualmente, oclusão completa da luz vascular por proliferação endarterial ou trombo. A terceira etapa é a granular nodular (EGN), na qual a vesícula tende a reduzir seu tamanho, tornando seu conteúdo semi-sólido. Nessa etapa, podemse visualizar restos do parasita, o escólex é transformado em um grânulo mineralizado e o infiltrado inflamatório é composto por células mononucleares. Na etapa final ou etapa nodular calcificada (ENC), os constituintes do parasita não são identificados, consistindo em um nódulo sólido, mineralizado, rodeado totalmente por tecido conjuntivo denso não modelado'12 2021242629 .

Os objetivos desse trabalho foram avaliar a prevalência da cisticercose, classificar as etapas evolutivas dos cisticercos localizados nos encéfalos e corações humanos, diferenciar essas etapas de acordo com os aspectos macro e microscópicos dos processos patológicos gerais e comparar os processos patológicos encontrados nos encéfalos e nos corações.

\section{MATERIAL E MÉTODOS}

Após aprovação pelo Comitê de Ética em Pesquisa da Faculdade de Medicina do Triângulo Mineiro (FMTM) foram revisados, retrospectivamente, protocolos de autópsias completas realizadas no Hospital Escola da FMTM, em Uberaba (MG), no período de 1970 a 2002.

Foram realizadas as análises macro e microscópica dos cisticercos, conforme critérios descritos na literatura 122021242629 . Essas análises foram sempre realizadas pelos mesmos observadores, aleatoriamente, e de acordo com um protocolo estabelecido previamente, que identificava os processos patológicos gerais que poderiam ocorrer em três zonas distintas: no próprio parasita, na interface hospedeiro-parasita e no tecido do hospedeiro ${ }^{31}$. Assim, os cisticercos foram classificados, de acordo com sua fase evolutiva, em EV, EVC, EGN e ENC e foram comparadas as alterações anátomo-patológicas tentando estabelecer uma associação macro e microscópica entre as diferentes etapas dos cisticercos.

Foram analisados 7 encéfalos e 10 corações fixados em formaldeído a $10 \%$, originados de indivíduos autopsiados no Hospital Escola da Faculdade de Medicina do Triângulo Mineiro, Uberaba, MG, no período de 1970 a 2000. Em cada órgão foi retirado um fragmento contendo o cisticerco, exceto em 2 encéfalos nos quais foram encontrados 2 cisticercos, totalizando 19 fragmentos com cisticercos. Em seguida, esses foram processados, rotineiramente, realizando a microtomia para a obtenção de cortes de $6 \mathrm{~mm}$ de espessura e corados segundo a técnica de Hematoxilina-Eosina. Em todas os casos além da Hematoxilina-Eosina foram coradas lâminas pelo Picro-sírius, para identificação de fibrose; pelo ácido periódico de Schiff (PAS), para depósitos de radicais glicídicos; Giemsa; Vermelho Congo, para beta-fibrilose; e nos cortes de coração também o Weigert-van Gieson, para fibras elásticas. Em um terço das lâminas, devido a suspeita de outros depósitos anormais ou para diferenciar estruturas, foram utilizadas as colorações von Kossa, para depósito de sais de cálcio; Hematoxilina Férrica, para identificação de estruturas constitucionais do parasita; Pearls, para depósito de ferro e a Prata Metenamina com o ácido periódico, para identificar a presença de fungos.

Os processos patológicos gerais analisados foram: 1) patologia da célula; 2) patologia do interstício; 3) alterações locais da circulação sangüínea; 4) pigmentações patológicas; 5) calcificação patológica; 6) edema; 7) processo inflamatório; 8) distúrbios do crescimento e da diferenciação celular.

As alterações microscópicas foram classificadas de forma semi-quantitativa, seguindo os seguintes critérios: ausente, discreta com comprometimento de até $25 \%$ da área, moderada de 26 a 50\% e acentuada acima de $50 \%{ }^{30}$.

Para a análise estatística foi elaborado um banco de dados eletrônico. Em seguida, as variáveis foram testadas para verificar se tinham distribuição normal, através do teste de Kolmogorov-Smirnov. Nos casos com distribuição normal utilizamos o teste "t" de Student. Nos casos em que a distribuição não foi normal, 
utilizamos o teste de Mann-Whitney. As proporções foram comparadas pelo teste do $\chi^{2}$, quando necessário acompanhado do Teste Exato de Fisher. As diferenças foram consideradas estatisticamente significantes quando a probabilidade de rejeição da hipótese de nulidade foi menor que $5 \%(p<0,05)$.

\section{RESULTADOS}

Foram revisados 2.639 protocolos de necropsias realizadas no período de 1970 a 2000. Desses, 421 foram excluídos por serem de indivíduos com idade abaixo de 15 anos e/ou por estarem incompletos. Dos 2.218 protocolos restantes, encontramos relatos de cisticercose em $71(3,2 \%)$ indivíduos, sendo que $18(25,3 \%)$ casos apresentavam cisticercose cardíaca e 53 (74,6\%) cisticercose encefálica, encontrando associação das duas formas somente em $2(2,8 \%)$ casos.

Para a análise das etapas evolutivas, foram selecionados 7 encéfalos e 10 corações, levando em consideração comparativamente os aspectos macro e microscópicos. Dessa forma, foi possível identificar e descrever as etapas evolutivas da cisticercose encefálica e cardíaca (Tabela 1).

$\mathrm{Na}$ cisticercose encefálica, encontraram-se cisticercos localizados no anterior de ventrículos na EV e EVC, meningo-cortical na EV, EVC, EGN e ENC, na substância branca na ENC e no hipotálamo na ENC. $\mathrm{Na}$ Tabela 2, pode-se verificar a distribuição dos processos patológicos gerais de acordo com a etapa evolutiva.

$\mathrm{Na}$ cisticercose cardíaca, foram encontrados cisticercos subepicárdicos na EV, EVC e EGN, intramiocárdicos na EVC e EGN, e subendocárdicos, na EVC, porém não foram encontrados cisticercos na ENC em nenhuma localização. Na Tabela 3, pode-se verificar a distribuição dos processos patológicos gerais de acordo com a etapa evolutiva. Não foi encontrada diferença estatisticamente significativa na comparação desses processos na cisticercose encefálica e cardíaca. Da mesma forma, não foram observadas diferenças significativas entre a classificação macro e microscópica das etapas evolutivas dos cisticercos .

Tabela 1 - Distribuição das etapas evolutivas avaliadas, macro e microscopicamente, nos 19 cisticercos localizados nos encéfalos e corações de indivíduos com cisticercose no período no período de 1970 a 2002.

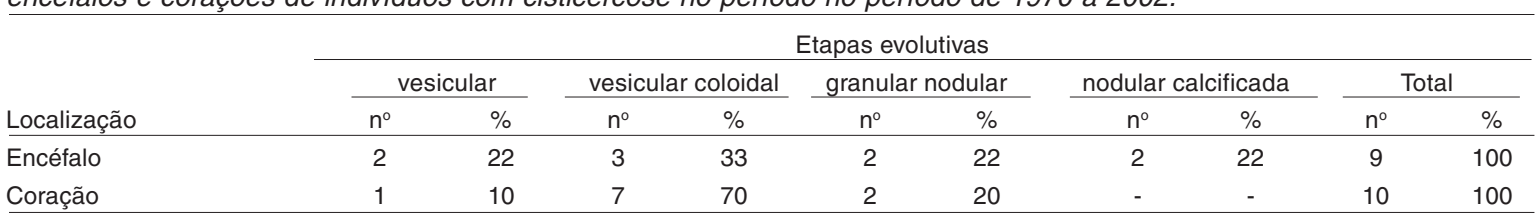

$\chi^{2}=3,891 ; p=0,273$

Tabela 2 - Distribuicão dos processos patológicos gerais de acordo com as etapas evolutivas em 9 fragmentos de 7 encéfalos de indivíduos com cisticercose no período de 1970 a 2002.

\begin{tabular}{|c|c|c|c|c|c|c|c|c|c|c|c|c|}
\hline \multirow[b]{3}{*}{ Processos Patológicos } & \multicolumn{12}{|c|}{ Etapas evolutivas } \\
\hline & \multicolumn{3}{|c|}{ vesicular } & \multicolumn{3}{|c|}{ vesicular coloidal } & \multicolumn{3}{|c|}{ granular nodular } & \multicolumn{3}{|c|}{ nodular calcificada } \\
\hline & paras & inter & hosp & paras & inter & hosp & paras & inter & hosp & paras & inter & hosp \\
\hline Necrose & & & + & & & & +++ & & & & & + \\
\hline Depósito de radical glicídico & +++ & + & & ++ & ++ & & ++ & & & + & + & \\
\hline Beta-fibrilose & & + & & & ++ & & +++ & +++ & & + & + & \\
\hline Fibrose & + & & & ++ & & & +++ & & & +++ & & \\
\hline Edema & & & + & & & ++ & & & +++ & & & + \\
\hline Calcificação & + & & & + & + & & + & + & & + & & \\
\hline Corpos amiláceos & & & + & & & & & & + & & & + \\
\hline Hemossiderose & & & & + & & & & & & + & & \\
\hline Inflamação & + & + & + & ++ & ++ & ++ & +++ & +++ & +++ & & + & + \\
\hline Hipotrofia de neurônios e células da glia & & & + & & & & & & & & & \\
\hline Gliose & & + & & & ++ & ++ & & +++ & +++ & & +++ & +++ \\
\hline Proliferação vascular & & + & + & & ++ & ++ & & +++ & +++ & & + & + \\
\hline
\end{tabular}

(paras) parasita, (inter) interface hospedeiro-parasita, (hosp) tecido do hospedeiro,

$(+)$ intensidade discreta, $(++)$ moderada e $(+++)$ acentuada. 
Tabela 3 - Distribuição dos processos patológicos gerais de acordo com as etapas evolutivas em 10 corações de indivíduos com cisticercose autopsiados no período de 1970 a 2000.

\begin{tabular}{|c|c|c|c|c|c|c|c|c|c|}
\hline \multirow[b]{3}{*}{ Processos patológicos } & \multicolumn{9}{|c|}{ Etapas Evolutivas } \\
\hline & \multicolumn{3}{|c|}{ Vesicular } & \multicolumn{3}{|c|}{ Vesicular coloidal } & \multicolumn{3}{|c|}{ Granular nodular } \\
\hline & paras & inter & hosp & paras & inter & hosp & paras & inter & hosp \\
\hline Necrose & & & & & & & & & ++ \\
\hline Degeneração hialina & & & & & + & + & & & ++ \\
\hline Degeneração hidrópica & & & + & & + & + & & & ++ \\
\hline Depósito de radical glicídico & +++ & +++ & & ++ & ++ & ++ & ++ & ++ & \\
\hline Beta-fibrilose & & + & & & ++ & & ++ & ++ & \\
\hline Fibroelastose & & & & & & ++ & & & \\
\hline Fibrose & + & + & + & + & ++ & ++ & & +++ & ++ \\
\hline Edema & & & + & & & ++ & & & ++ \\
\hline Calcificação & & & & & + & & & & \\
\hline \multicolumn{10}{|l|}{ Hemossiderose } \\
\hline Congestão & & + & & & ++ & ++ & & +++ & ++ \\
\hline Inflamação & & + & + & & ++ & ++ & +++ & +++ & ++ \\
\hline Proliferação vascular & & + & + & & ++ & ++ & & +++ & ++ \\
\hline
\end{tabular}

(paras) parasita, (inter) interface hospedeiro-parasita, (hosp) tecido do hospedeiro

$(+)$ intensidade discreta, $(++)$ moderada e $(+++)$ acentuada.

\section{DISCUSSÃO}

Neste estudo, verificou-se a ocorrência de 3,2\% de cisticercose em autópsias. Esses dados estão de acordo com outros estudos, cuja ocorrência variou de $0,12 \%$ a $9 \%{ }^{157815}$ e em outro estudo que realizamos também com material de autópsias, cuja ocorrência de cisticercose foi de $3,3 \%{ }^{19}$. Porém, são superiores aos descritos na região do Triângulo Mineiro, como os 2,4\% de Gobbi e cols ${ }^{15}$ e os $1,4 \%$ de Costa-Cruz e cols ${ }^{8}$. Esse fato ocorreu, talvez, porque os estudos tenham sido realizados em diferentes épocas e com distintos procedimentos metodológicos.

Na ENC, com a desintegração do parasita, a reação inflamatória tinha tendência a se reduzir persistindo as células gigantes, englobadas na cápsula fibrosa, com gliose e edema. Esse fato, segundo Márquez-Monter ${ }^{20}$, indicaria uma continuidade na reação do hospedeiro contra os restos do parasita sem, contudo, apresentar uma associação com o tipo ou a intensidade da resposta inflamatória.

Na cisticercose encefálica, além dessas características já descritas por vários autores ${ }^{3} 42202429$, no presente estudo, encontramos processos patológicos que merecem destaque, tais como: necrose, depósito de radicais glicídicos, beta-fibrilose, calcificação, alteração no crescimento e diferenciação celular.

A necrose foi detectada nesse estudo, principalmente, nas etapas EVC e EGN, isto é, nas fases em que o parasita está sofrendo maior ação da resposta imunológica do hospedeiro, estando em concordância com Pittella ${ }^{24}$.

Os depósitos de radicais glicídicos estiveram presentes em todas as fases evolutivas dos cisticercos analisados nesse estudo, porém, foram vistos com maior intensidade nas etapas EV e EVC, acometendo o parasita e a interface hospedeiro-parasita. Esses dados estão de acordo com Thomas e cols ${ }^{32}$, que correlacionaram a presença desses depósitos no interior de vacúolos intraparasitários com o metabolismo do parasita.

A beta-fibrilose (amiloidose) esteve presente em todas as fases evolutivas, porém, foi vista com maior freqüência e intensidade nas EVC e EGN. De acordo com Cotran e cols $^{9}$, esse tipo de depósito ocorre em outras doenças devido a complicações de um processo inflamatório crônico ou de processo que destrói o tecido, sendo, portanto, chamado de beta-fibrilose secundária. Embora na literatura não haja relatos anteriores sobre esse processo patológico, sabe-se que várias substâncias, geralmente de natureza protéica, podem sofrer transformação e se depositarem como beta-fibrilas, dentre elas as imunoglobulinas e as citocinas. Rodrigues Jr e cols ${ }^{27}$ demonstraram elevados níveis de citocinas no líquor de pacientes com neurocisticercose. Assim, conclui-se que pelo menos parte das beta-fibrilas encontradas possa estar relacionada à elevação dos níveis de interleucinas.

Diferentemente de outros trabalhos, a calcificação patológica esteve presente, com intensidade discreta, nas etapas iniciais de evolução do cisticerco, EV e EVC. Esses achados diferem de relatos anteriores que descrevem a calcificação como um processo marcante na cisticercose ${ }^{33}$, principalmente na etapa evolutiva mais avançada, sendo considerado o achado mais importante para o diagnóstico através da tomografia computadorizada ${ }^{24}$.

O infiltrado inflamatório foi encontrado em intensidade variável em todas as etapas evolutivas, particularmente nas EVC e EGN, estando de acordo com o descrito na literatura ${ }^{20}$.

Em relação à cisticercose cardíaca, com exceção do infiltrado inflamatório, as informações obtidas em outros trabalhos são escassas, variando desde ausência de 
inflamação, até miocardite e insuficiência cardíaca congestiva ${ }^{172025}$.

Outro achado aparentemente inédito no presente trabalho foi a associação da fibroelastose, particularmente, com a cisticercose endocárdica. A etiologia imputada a este processo é variada, destacando-se aquelas primárias, ligadas à herança genético-cromossômica, ou a doenças infecciosas e parasitárias ${ }^{2616}$. Dependendo da intensidade, cursa com sintomatologia, podendo até estar associada à causa de morte ${ }^{10}{ }^{18}$. Nos casos do presente estudo, a fibroelastose esteve restrita às adjacências do cisticerco e, aparentemente, em nenhum dos casos teve relação direta com a causa de morte.
A maioria dos trabalhos que descrevem as etapas evolutivas da cisticercose restringe-se quase exclusivamente aos aspectos macroscópicos ${ }^{12} 2025$. No entanto, no presente trabalho, verificamos que é possível a confrontação do diagnóstico macroscópico dos processos patológicos gerais com as características microscópicas desses mesmos processos em cada etapa evolutiva.

Em conclusão, no presente trabalho, demonstrouse que a classificação nas etapas evolutivas da cisticercose, baseada nos processos patológicos gerais, é similar tanto macro quanto microscopicamente. Além disso, essa classificação pode ser aplicada tanto na cisticercose humana encefálica como na cardíaca.

\section{AGRADECIMENTOS}

Os autores agradecem aos funcionários da Disciplina de Patologia Geral da Faculdade de Medicina do Triângulo Mineiro, Vandair Gonçalves Pereira, Aloísio Costa, Elenemar Flausino Borges, Maria Aparecida da Silva Dias, pelo apoio técnico na realização do trabalho.

\section{REFERÊNCIAS BIBLIOGRÁFICAS}

1. Agapejev S. Epidemiology of neurocysticercosis in Brazil. Revista do Instituto de Medicina Tropical de São Paulo 38:207-216, 1996.

2. Almeida $\mathrm{HO}$, Chapadeiro E. Alterações endocárdicas na região apical de corações chagásicos crônicos. Revista do Instituto de Medicina Tropical de São Paulo 20:293-299, 1978.

3. Amatya BM., Kimula Y. Cysticercosis in Nepal. A histopathologic study of sixty-two cases. The American Journal of Surgical Pathology 23:1276-1279, 1999.

4. Barbosa AP, Costa-Cruz JM, Almeida S, Campos DMB. Cisticercose: Fatores relacionados à interação parasitohospedeiro, diagnóstico e soroprevalência. Revista de Patologia Tropical 29:17-34, 2000

5. Briceño CE, Biagi F, Martinez B. Cisticercosis. Observaciones sobre 97 casos de autopsia. Prensa Médica de México 26:193-197, 1961

6. Cary NR, Fox B, Wright DJ, Cutler SJ, Shapiro LM, Grace AA. Fatal Lyme carditis and endodermal heterotopia of the atrioventricular node. Postgraduate Medical Journal 66:134-136, 1990.

7. Chimelli L, Lovalho AF, Takayanagui OM. Neurocisticercose. Contribuição da necropsia na consolidação da notificação compulsória em Ribeirão Preto-SP. Arquivos de Neuropsiquiatria 56:577-584, 1998.

8. Costa-Cruz JM, Rocha A, Silva AM, Moraes AT, Guimarães AHB, Salomão EC, Alcântara TM. Ocorrência de cisticercose em necropsias realizadas em Uberlândia, Minas Gerais, Brasil. Arquivos de Neuropsiquiatria 53:227-232, 1995.

9. Cotran RS, Kumar V, Robbins SL, Schoen FJ. Doenças da imunidade. In: Cotran RS, Kumar V, Robbins SL, Schoen FJ (ed) Patologia estrutural e Funcional. 5 $5^{\text {a }}$ edição, Guanabara Koogan, Rio de Janeiro, p. 152-212,1996.

10. De Letter EA, Piette MHA. Endocardial fibroelastosis as a cause of sudden unexpected death. American Journal of Forensic Medicine and Pathology 20:357-363, 1999.

11. Del Brutto $\mathrm{OH}$, Sotelo J. Neurocysticercosis: an update. Reviews of Infectious Diseases 10:1075-1087, 1988.
12. Escobar-Izquierdo A. La patologia de la neurocisticercosis. Gaceta Medica de Mexico 124:202-206, 1988.

13. Estañol B. Controversias em cisticercosis cerebral. Gaceta Medica de Mexico 119:461-465, 1983.

14. Flisser A. Taeniasis-cysticercosis: an introduction. Southeast Asian Journal of Tropical Medicine and Public Health 22:233-235, 1991.

15. Gobbi H, Adad SJ, Neves RR, Almeida HO. Ocorrência de cisticercose (Cysticercus cellulosae) em pacientes necropsiados em Uberaba, MG. Revista de Patologia Tropical 9:51-59, 1980.

16. Hilton DA, Variend S, Pringle JH. Demonstration of Coxsackievirus RNA in formalin-fixed tissue-sections from childhood myocarditis cases by in situ hybridization and the polymerase chain-reaction. Journal of Pathology 170:45-51, 1993.

17. Ibarra-Perez Cl, Diez JF, Trujillo FR. Myocardial Cysticercosis. Southern Medical Journal 65:484-486, 1972.

18. Lane KL, Herzberg AJ, Reimer KA, Bradford WD, Schall SA. Endocardial fibroelastosis with coronary-artery thromboembolism and myocardial-infarction. Clinical Pediatrics 30:593-598, 1991.

19. Lino Júnior RS, Reis MA, Teixeira VPA. Ocorrência de cisticercose (Cysticercus cellulosae) encefálica e cardíaca em necropsias. Revista de Saúde Pública 33:60-63, 1999.

20. Marquéz-Monter H. Patologia de la cisticercosis. Gaceta Medica de Mexico 103:230-242, 1972.

21. Montemór Netto MR, Gasparetto EL, Faoro LN, Reis Filho JS, De Toni GS, Carvalho Neto, A, Torres LFB. Estudo clínico e patológico de 27 casos de necropsia. Arquivos de Neuropsiquiatria 58:883-889, 2000.

22. Nascimento E. Teníase e cisticercose. In: Neves DP (ed)

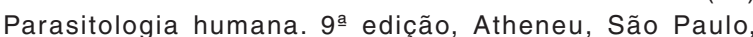
p. 244-256, 1998.

23. Pedretti L, Bedaque EA, Sotelo J, Del Brutto OH. Cisticercose. In: Veronesi R, Focaccia R (eds) Tratado de Infectologia, Atheneu, São Paulo, p. 1332-1347, 1999 
24. Pittella JEH. Neurocysticercosis. Brain Pathology 4:681-693, 1997.

25. Prabhakar BR, Manjari M, Vadehra PL. Cardiac cysticercosis. Indian Journal of Pathology and Microbiology 33:377-378, 1990.

26. Robinson P, Atmar RL, Lewis DE, White AC. Granuloma cytokines in murine cysticercosis. Infection and Immunity 65:2925-2931, 1997.

27. Rodrigues Jr V, Mello FA, Magalhães EP, Ribeiro SBF, Marquez JO. Interleukin-5 and interleukin-10 the major cytokines in cerebrospinal fluid from patients with active neurocysticercosis. Brazilian Journal of Medical and Biological Research 33:10591063, 2000.

28. Shandera WX, White Jr AC, Chen JC, Diaz P, Armstrong R. Neurocysticercosis in Houston, Texas: report of 112 cases. Medicine 73:37-52, 1994.

29. Sotelo J, Del Brutto O. Brain cysticercosis. Archives of Medical Research 31:3-14, 2000.
30. Teixeira VPA, Hial V, Gomes RAS, Castro ECC, Reis MG, Rodrigues MLP, Guimarães JV, Reis MA. Correlation between adrenal central vein parasitism and heart fibrosis in chronic chagasic myocarditis. American Journal of Tropical Medicine and Hygiene 56:177-180, 1997.

31. Thomas JA, Knoth R, Schwechheimer K, Volk B. Disseminated human neurocysticercosis. Acta Neuropathologica 78:594-604, 1989.

32. Thomas JA, Kothare SN, Baptist SJ. Cysticercus cellulosae. The Journal of Tropical Medicine and Hygiene 76:106-110, 1973.

33. Vargas-Parada L, Merchant MT, Willms K, Laclette JP. Formation of calcareous corpuscles in the lumen of excretory canals of Taenia solium cysticerci. Parasitology Research 85:88-92, 1999.

34. White Jr AC. Neurocysticercosis: updates on epidemiology, pathogenesis, diagnosis, and management. Annual Review of Medicine 51:187-206, 2000. 